\title{
METAIS TRAÇO EM PEIXES E FILTRADORES EM QUATRO ESTUÁRIOS DA COSTA BRASILEIRA
}

\author{
Luis Felipe NIENCHESKI \\ Eunice da Costa MACHADO \\ Ieda Maria Oliveira SILVEIRA \\ Manuel de Jesus FLORES MONTES
}

Recebido em: $12 / 08 / 2013$
Aceito em: 06/06/2014

\section{ABSTRACT}

The Brazilian coast is little studied on bioaccumulation of trace metal aimed to assess the status of contamination in fish and filter feeding organisms. The objective of this work is to obtain a diagnosis of four estuaries of social and economic importance, to be subsequently submitted to further analysis, to search for the cause and effect relationship between biodiversity and natural variations of human actions developed on the coast of Pernambuco, Espírito Santo, Paraná and Rio Grande do Sul State. Some

metals exhibited concentrations that exceeded the maximum permitted by Brazilian legislation, revealing the urgent need for a systematic study on bioaccumulation of trace metal and their chemical speciation. This work is a pilot study that provides background information and, establishes the basis for a monitoring program: it promoted staff training, standardization of analytical procedures and established a model for future monitoring programs.

Keywords: bioindicators, trace metal, Brazilian estuaries.

\section{RESUMO}

$\mathrm{Na}$ costa brasileira são escassos os estudos sobre bioacumulação de metais traço que visam acessar o status de contaminação em peixes e organismos filtradores. O objetivo deste trabalho é obter um diagnóstico de quatro estuários de importância social e econômica, passiveis de serem a posteriori submetidos a analise mais aprofundada, para a busca da relação de causa e efeito entre variações naturais da biodiversidade e as ações antrópicas desenvolvidas na costa de Pernambuco, Espírito Santo, Paraná e Rio Grande do Sul.
Alguns metais exibiram concentrações que excederam os máximos permitidos pela legislação, revelando a necessidade urgente de um estudo sistemático sobre bioacumulação de metais traço e a forma química que se apresentam. Esse trabalho lança as bases para um programa de monitoramento, pois se trata de um estudo piloto disponibilizando informações de base, que promoveu treinamento de equipes $\mathrm{e}$ padronização de procedimentos analíticos e, fornecendo um modelo para programas futuros.

Palavras - chave: bioindicadores, metais traço, estuários brasileiros.

\section{INTRODUÇÃO}

A questão relacionada à contaminação e poluição dos ambientes aquáticos tem sido uma crescente preocupação, não somente graças às frequentes ocorrências de inúmeros compostos inorgânicos e orgânicos contendo metais traço, nos compartimentos biótico e abiótico, mas principalmente pelos seus efeitos deletérios à biota dos ecossistemas aquáticos quando em concentrações anormais. Deste modo, é importante avaliar a distribuição destes contaminantes e também a sua biodisponibilidade.

A avaliação das concentrações de metais pesados constitui-se em uma preocupação atual muito enfatizada, principalmente porque sua persistência no ambiente perturba processos biológicos, em função de sua acumulação e alta toxicidade para determinados processos

\footnotetext{
${ }^{1}$ Instituto de Oceanografia, Universidade Federal do Rio Grande (IO-FURG). felipeniencheski@furg.br

${ }^{2}$ Centro de Estudos do Mar, Universidade Federal do Paraná (CEM-UFPr). eunice.ufpr@gmail.com

${ }^{3}$ Departamento de Oceanografia e Ecologia, Universidade Federal Fluminense (DOC-UFES). CEPEMAR

Serviços de Consultoria em Meio Ambiente Ltda. iedamaria.oliveiradasilveira@gmail.com

${ }^{4}$ Departamento de Oceanografia, Universidade Federal de Pernambuco (DOC-UFPE). manuel@ufpe.br
} 
NIENCHESKI, Luis Felipe, et al. Metais traço em peixes e filtradores em quatro estuários da costa brasileira.

metabólicos, bem como outros efeitos adversos que os metais podem causar sobre os ecossistemas aquáticos.

A biota é um dos compartimentos mais utilizados para monitorar o ambiente, pois integra a contaminação ao longo do tempo. Embora existam estudos sobre bioacumulação de metais traço em algumas regiões da costa brasileira, escassos são aqueles que visam acessar o status de contaminação por elementos traço em peixes e organismos filtradores em diversas regiões do litoral brasileiro, marcado por diferentes ecossistemas (manguezais, dunas, ilhas, costões, baías, restingas, falésias e recifes de corais), e que abrigam enorme variedade de espécies. Muitas dessas áreas estão ameaçadas por graves processos de degradação, como a exploração excessiva dos recursos pesqueiros, ocupação desordenada do litoral, entre outros fatores.

O objetivo deste trabalho é obter um diagnóstico de 4 estuários de importância social e econômica, passiveis de serem a posteriori submetidos à analise mais aprofundada, para a busca da relação de causa e efeito entre variações naturais da biodiversidade e as ações antrópicas desenvolvidas nas regiões costeiras dos estados de Pernambuco, Espírito Santo, Paraná e Rio Grande do Sul. O conhecimento gerado dará subsídio para a solução de desafios semelhantes em outras áreas do litoral.

Neste trabalho, a bioacumulação de metais em peixes será comparada vis-a-vis à legislação brasileira, quanto às concentrações máximas permitidas para consumo humano e, a bioacumulação em cracas refletirá a qualidade do material em suspensão, em áreas consideradas impactadas e outras longe das fontes diretas de contaminação, para cada ambiente estudado.

\section{MATERIAL E METODOS}

\section{Locais de coleta:}

\section{Rio Grande}

Ao sul da Lagoa dos Patos forma-se o estuário, que representa cerca da décima parte dessa Lagoa. Neste, as diversas enseadas existentes ocupam aproximadamente $28 \%$ da área total estuarina. Este estudo foi desenvolvido nas enseadas Saco da Mangueira e Saco do Arraial, situadas ao sul do estuário $\left(32^{\circ} 00^{\prime}, 32^{\circ} 10^{\prime} \mathrm{S}\right.$ e $\left.52^{\circ} 02^{\prime}, 5^{\circ} 15^{\prime}\right)$. O Saco da Mangueira posiciona-se ao sul da cidade do Rio Grande (cerca de 180.00 hab.).

A contaminação de suas águas que margeiam a cidade do Rio Grande é causada basicamente pelos lançamentos de efluentes domésticos e industriais sem tratamento adequado, oficiais e clandestinos, oriundos da cidade do Rio Grande e do Distrito Industrial. O somatório de todos esses efluentes resulta em forte eutrofização, devido à alta concentração de nutrientes, apesar de ser uma região de intensa pesca artesanal (Baumgarten et al. 1995, 2001).

Na margem leste do Saco da Mangueira não há ocupação urbana, mas situa-se parte de um Distrito Industrial, onde estão instaladas duas indústrias de fertilizantes e duas de processamento de soja e extração de óleo.

Baumgarten et al. (2001) identificaram no Saco da Mangueira basicamente três áreas com características hidroquímicas diferentes em função de diversificadas fontes de poluição por matéria orgânica, sendo uma área lateral à cidade, outra lateral ao Distrito Industrial e a terceira, bem ao fundo dessa enseada, onde os aportes antrópicos são mais escassos e há chegada de águas continentais.

O Saco do Arraial foi o outro local estudado. Essa enseada situa-se na direção oeste de Rio Grande, relativamente afastada da área urbana, sendo, portanto, mais protegida de aportes antrópicos urbanos e industriais, embora em suas margens sejam desenvolvidas algumas atividades agrícolas de pequeno e médio porte. Trata-se de uma área bem mais aberta do que o Saco da Mangueira e, assim, com maior renovação de água. Apresenta um formato de ferradura, onde no centro situa-se a Ilha dos Marinheiros. Isso faz com que haja duas aberturas de comunicação dessa enseada com o resto do estuário. Por esses aspectos, foi considerada nesse trabalho como sendo área "controle". 
NIENCHESKI, Luis Felipe, et al. Metais traço em peixes e filtradores em quatro estuários da costa brasileira.

\section{Paraná}

O Complexo Estuarino de Paranaguá situa-se na costa sul do Brasil ( $25^{\circ} 30^{\prime} \mathrm{S}, 48^{\circ} 30^{\prime} \mathrm{W}$ ) e é formado pelas baías de Antonina, Paranaguá, Laranjeiras e Pinheiros. É um sistema onde áreas prístinas de mangue ainda são encontradas e as atividades de pesca e aquicultura são importantes economicamente (Liebzeit et al. 2011). Apesar da importância ecológica e econômica do Complexo Estuarino de Paranaguá, este tem sido influenciado por agentes antrópicos de diferentes fontes, associadas às atividades portuárias, indústrias de fertilizantes e esgotos domésticos (Martins et al. 2010), especialmente na região de Paranaguá, com maior índice demográfico do litoral paranaense (IBGE, 2010).

Um pequeno número de estudos foi realizado com contaminantes ambientais na região, alguns deles identificam a contaminação dos sedimentos por hidrocarbonetos policíclicos aromáticos (Fillmann et al. 2007; Froehner et al. 2011), e por elementos-traço, tais como As, $\mathrm{Ni}, \mathrm{Zn}$ e Cu (Sá et al. 2006; Choueri et al. 2009, Martins et al. 2012), além de elementos traço em suas formas mais biodisponíveis (Sá 2003). Outro estudo realizado com a biota da região também aponta a contaminação de As, $\mathrm{Cu}$ e $\mathrm{Zn}$ em ostras Crassostrea rhizophorae (Castello 2010). Angeli (2012) analisou sete elementos traço (As, Cd, $\mathrm{Cu}, \mathrm{Cr}, \mathrm{Ni}, \mathrm{Pb}$ e $\mathrm{Zn}$ ) no bagre amarelo (Cathorops spixii) (Agassiz) e o bagre urutu (Genidens genidens) (Valenciennes) do Complexo Estuarino de Paranaguá e, de acordo com a Agência Nacional de Vigilância SanitáriaANVISA (1998), os níveis de $\mathrm{Cr}$ e As excederam os limites permitidos.

\section{Espírito Santo}

O estuário dos rios Piraquê-Açu e Piraquê-Mirim está localizado no distrito de Santa Cruz, município de Aracruz (ES), ao norte do estado, distando aproximadamente $50 \mathrm{~km}$ de Vitória, a capital do Espírito Santo. O estuário está localizado próximo às coordenadas $40^{\circ} 09^{\prime}$ W e $19^{\circ} 5^{\prime}$ $\mathrm{S}$, aproximadamente $2 \mathrm{~km}$ a montante da foz os dois rios se encontram, fazendo com que o sistema tenha forma de "Y". O estuário (com 510 ha de espelho d'água) faz parte do projeto de gerenciamento costeiro do estado do Espírito Santo, setor Litoral Norte, abrangido pela Zona de Proteção Ambiental I (ZPA I) (SEAMA, 1998). A área de manguezal possui aproximadamente $12,3 \mathrm{~km}^{2}$ (Barroso, 2004) e a floresta é composta basicamente por Rhizophora mangle (mangue vermelho), Laguncularia racemosa (mangue branco) e Avicennia schaueriana (mangue preto).

É uma área de concentração de grandes indústrias, incentivada pela política estadual de implantação de indústrias de grande porte, como a indústria de celulose. Os principais vetores de pressão antrópica que ameaçam a integridade deste ecossistema estão associados aos diversos usos do solo na bacia hidrográfica, tais como a exploração intensa e predatória dos seus recursos florestais, o reflorestamento homogêneo de eucalipto, e outros empreendimentos agrícolas do setor agroindustrial em desenvolvimento; a pressão imobiliária bem como a supressão das áreas de manguezais para diversos fins; a operação de embarcações entre outras atividades ligadas à navegação; despejo de efluentes domésticos; e riscos ambientais associados à instalação portuária da Portocel, tais como derramamento de petróleo e de cargas tóxicas, localizada na área costeira circunvizinha.

Embora estejam interligados, assumiu-se que o rio Piraquê-Mirim poderia ser usado como ambiente controle, porque, assim como o Saco do Arraial em Rio Grande, é mais afetado por atividade agrícola, enquanto o rio Piraquê-Açu poderia ser usado como indicador de ambiente poluído, já que recebe a maior parte das demais influências antrópicas citadas anterioriormente.

\section{Pernambuco}

O Litoral Norte de Pernambuco abrange importantes áreas estuarinas, que são formadas por diferentes rios de pequeno porte, entre eles o Botafogo e o Carrapicho/Itapessoca, que

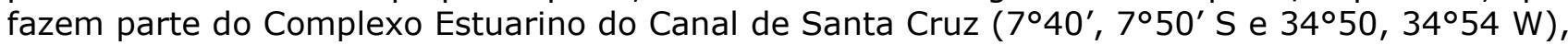
dentro da APA de Santa Cruz. Estas áreas abrangem cerca de 15.675 ha, sendo a área de manguezal em torno de 8.000 ha. Esta região é caracterizada por ter um clima tropical, com temperatura do ar indo de $20^{\circ} \mathrm{C}$ a $34^{\circ} \mathrm{C}$, com uma compensação média de $27^{\circ} \mathrm{C}$. O Canal de Santa Cruz, que separa a ilha da costa, possui uma extensão de $22 \mathrm{~km}$, e larguras variáveis de 0,6 a 1,5 km. Toda a área é muito rasa, com profundidades variando, na baixa-mar, em torno 
NIENCHESKI, Luis Felipe, et al. Metais traço em peixes e filtradores em quatro estuários da costa brasileira.

dos 4-5 m. Este canal se comunica ao norte com o mar pela Barra Catuama, e ao sul, pela Barra Orange, podendo a profundidade atingir nestes locais até $10 \mathrm{~m}$ ou mais, sendo as partes mais sensíveis à ação das marés, que são do tipo mesomarés e semi-diurnas.

Segundo a Agência Estadual de Meio Ambiente-CPRH (2004) na bacia de drenagem do Complexo Estuarino do Canal de Santa Cruz, com área total de $730 \mathrm{~km}^{2}$, estão localizadas varias indústrias dentre as quais se destacam: as metalúrgicas, químicas, têxtil, de beneficiamento de alimentos e fibra de coco, de produção de papeis, plásticos, tintas e cimento. Este Complexo Estuarino é um dos mais produtivos do Estado de Pernambuco, abastecendo o Grande Recife e adjacências, com peixes, moluscos e crustáceos.

A bacia do rio Botafogo é a mais extensa dentre aquelas totalmente inseridas no Litoral Norte, ocupando o equivalente a 34,8\% desse setor litorâneo. Localiza-se nessa bacia, o único reservatório do Litoral Norte, integrado ao sistema de abastecimento da Região Metropolitana do Recife, a Barragem do Botafogo com capacidade para armazenar 27,5 milhões de $\mathrm{m}^{3}$. Os recursos naturais apresentam-se em progressiva deterioração devido ao destino inadequado do lixo, pesca predatória, desmatamento do manguezal para fins imobiliário e para a carcinicultura, e poluição proveniente de efluentes domésticos e industriais. As indústrias instaladas são consideradas potencialmente poluidoras, particularmente no que se refere a metais traço.

\section{Amostras}

Organismos amostrados: visando estabelecer as relações entre as concentrações dos principais indicadores químicos da contaminação ambiental com a biota representativa dos principais elos da cadeia trófica, foram analisados peixes Micropogonias furnieri (Rio Grande do Sul), Cathorops spixii (Paraná), Lutjanus synagris (Espirito Santo) e Bagre marinus (Pernambuco) e, organismos filtradores como a craca (Balanus improvisus) no Rio Grande e ostra (Crassostrea rhizophorae) no Paraná, Espírito Santo e Pernambuco.

\section{Tecidos biológicos analisados}

-peixe - foram retirados o fígado e porções do músculo.

-filtradores - foi extraída toda parte mole do organismo.

\section{Estações de coleta e frequência temporal das amostragens}

A estratégia amostral foi montada com base na filosofia do grupo temático "Qualidade Ambiental e Biodiversidade" (QA\&B), do Projeto "Uso e apropriação dos recursos costeiros" Instituto do Milênio, visando atender aos problemas de gestão costeira (www.mileniodomar.org.br).

Em cada uma das regiões de estudo foi escolhido um local de coleta distante de possíveis fontes de contaminação (referência) e, outro local com níveis de contaminação a priori assumidos como reduzidos. Para a escolha destes locais foi levada em consideração a distancia em relação a fontes antrópicas potencialmente poluidoras, tais como indústrias, portos e centros urbanos.

Para os peixes, tendo em vista a sua mobilidade, foi efetuada apenas uma coleta no local referência e outra no local poluído. Para os demais organismos, por serem sésseis, foram efetuadas mais de uma coleta ao longo de cada um desses locais.

Foram realizadas duas coletas com periodicidade semestral, sendo uma no verão e outra no inverno.

\section{Acondicionamento, transporte e processamento do material coletado.}

Os peixes, cracas e ostras foram lavados com água destilada e a seguir, foram retirados os tecidos biológicos seguindo as recomendações apresentadas em UNEP (1984a e b) e Avaliação Ambiental de Estuários Brasileiros: Diretrizes Metodológicas (2006). Os tecidos biológicos foram acondicionados em frascos plásticos previamente lavados com ácido e enxaguados com água Milli- $\mathrm{Q}^{\mathrm{TM}}$. Foram congelados $\mathrm{e}$, transportados dessa forma até 0 Laboratório de Hidroquimica (FURG). 
NIENCHESKI, Luis Felipe, et al. Metais traço em peixes e filtradores em quatro estuários da costa brasileira.

Após, foram liofilizadas (liofinizador Edwards Micro Modulyo). O tecido seco foi homogeneizado em um misturador plástico. A estocagem posterior foi feita em recipientes de plástico hermético e mantidos a $4^{\circ} \mathrm{C}$.

Os reagentes usados foram da categoria Suprapur ${ }^{\mathrm{TM}}$. Durante todo o procedimento foi usada água Milli-Q ${ }^{\mathrm{TM}}$. A vidraria foi submetida a um cuidadoso procedimento de limpeza, segundo M.E.S.L. (1997). A manipulação das amostras foi feita em uma capela de fluxo laminar.

\section{Procedimentos analíticos:}

\section{Digestão das amostras}

Aproximadamente $0,5 \mathrm{~g}$ de cada amostra foi digerida em $5 \mathrm{~mL}$ de $\mathrm{HNO}_{3}$ em recipiente (becker) de Teflon $^{\mathrm{TM}}$ com tampa e, transferido para um frasco de $25 \mathrm{~mL}$. Previamente para a determinação de $\mathrm{Hg}$, uma alíquota da amostra digerida foi tratada com $\mathrm{BrCl}$ (M.E.S.L, 1997).

\section{Procedimento de análise dos metais.}

Um sistema de vaporização a frio construído no Laboratório de Hidroquímica, acoplado com um AAS CG-AA 7000 foi usado para a determinação de $\mathrm{Hg}$. Detalhes deste sistema podem ser encontrados em Ustra (2001). Para determinar $\mathrm{Zn}$ e $\mathrm{Cu}$ foi utilizada chama de $\operatorname{ar}-\mathrm{C}_{2} \mathrm{H}_{2}$, usando um espectrofotômetro de absorção atômica CG-AA 7000. Outro espectrofotômetro, um ZEISS EA-AAS5 com atomização eletrotérmica, foi empregado para a determinação de $\mathrm{Cd}, \mathrm{Pb}$ e Cr. Os resultados foram obtidos segundo recomendações do M.E.S.L. (1997).

A precisão dos resultados analíticos foi conferida pela análise de material de referência Tuna 350, proveniente do "Marine Environmental Studies Laboratory" (M.E.S.L.), da Comissão Internacional de Energia Atômica (IAEA), com sede em Mônaco. Os resultados obtidos para a amostra de referência estavam de acordo (+/-8\%) com os valores certificados.

\section{RESULTADOS E DISCUSSÃO}

Os organismos aquáticos tendem a acumular metais pesados e contaminantes orgânicos em seus tecidos, mesmo quando a água possui níveis desses compostos abaixo da concentração máxima tolerada pela legislação, o que leva a grandes riscos de contaminação dentro da cadeia trófica. No caso de peixes, a ingestão de alimentos e de água é a principal rota de entrada de contaminantes nesses organismos, os quais se alojam diferentemente em cada tecido, baseado em suas características e em função dos processos bioquímicos internos do indivíduo. Animais filtradores como ostras e cracas filtram vários litros de água por hora e, consequentemente, podem concentrar de 10 a $10^{5}$ vezes vários contaminantes em seus tecidos, com relação à água do mar.

De acordo com a portaria $n^{\circ} 34 / 1984$ estabelecida pela DINAL (Divisão Nacional de Vigilância Sanitária), citada no ABIA (1991), as concentrações máximas de $\mathrm{Cu}, \mathrm{Cr}, \mathrm{Cd}, \mathrm{Pb}, \mathrm{Hg}$ e Zn permitidas nos alimentos utilizados para consumo humano são de $30 ; 0,10 ; 1,0 ; 2,0 ; 0,5$ e $50,0 \mathrm{mg} / \mathrm{kg}$ (peso úmido), respectivamente. Esses valores máximos estabelecidos pela legislação brasileira são expressos em peso úmido, enquanto os resultados desse trabalho são apresentados em peso seco. Para fins de comparação, as Tabelas 1 e 2 apresentam os valores da legislação foram transformados em peso seco, usando para tal um valor de umidade de $80 \%$, pois este é um valor médio do teor de água contida nos organismos estudados.

\section{Peixes}

Chumbo: todas as concentrações encontraram-se em conformidade com a legislação.

Cádmio: somente uma amostra de fígado, no inverno (seco), na Baía de Paranaguá, apresentou um valor ligeiramente acima do limite máximo. Embora deva ser considerado que o fígado não é usado para consumo humano direto (o que preconiza a legislação), esta concentração pode ser magnificada ao longo da cadeia trófica. 
NIENCHESKI, Luis Felipe, et al. Metais traço em peixes e filtradores em quatro estuários da costa brasileira.

Tabela 1 - Concentrações de metais traço obtidas em Agosto de 2003 e Fevereiro de 2004 em músculo e fígado de peixes: Micropogonias furnieri (Rio Grande do Sul), Cathorops spixii (Paraná), Lutjanus synagris (Espirito Santo) e Bagre marinus (Pernambuco).

\begin{tabular}{|c|c|c|c|c|c|c|c|c|c|c|c|c|c|c|}
\hline \multicolumn{3}{|c|}{ PEIXES } & Ago 2003 & Fev 2004 & $\begin{array}{c}\text { Ago } \\
2003\end{array}$ & $\begin{array}{c}\text { Fev } \\
2004\end{array}$ & $\begin{array}{l}\text { Ago } \\
2003\end{array}$ & $\begin{array}{c}\text { Fev } \\
2004\end{array}$ & $\begin{array}{c}\text { Ago } \\
2003\end{array}$ & $\begin{array}{c}\text { Fev } \\
2004\end{array}$ & $\begin{array}{c}\text { Ago } \\
2003\end{array}$ & $\begin{array}{c}\text { Fev } \\
2004\end{array}$ & $\begin{array}{l}\text { Ago } \\
2003\end{array}$ & $\begin{array}{c}\text { Fev } \\
2004\end{array}$ \\
\hline & PONTO & TECIDO & $\begin{array}{c}{[\mathrm{Hg}] \mathbf{n g} / \mathbf{g}} \\
\mathrm{ppb}\end{array}$ & $\begin{array}{c}{[\mathrm{Hg}] \mathbf{n g} / \mathbf{g}} \\
\mathrm{ppb}\end{array}$ & $\begin{array}{l}{[\mathrm{Zn}]} \\
\mu \mathrm{g} / \mathrm{g} \\
\mathrm{ppm}\end{array}$ & $\begin{array}{l}{[\mathrm{Zn}]} \\
\mu \mathrm{g} / \mathrm{g} \\
\mathrm{ppm}\end{array}$ & $\begin{array}{l}\text { [Cu] } \\
\mu \mathrm{g} / \mathrm{g} \\
\mathrm{ppm}\end{array}$ & $\begin{array}{l}\text { [Cu] } \\
\mu \mathrm{g} / \mathrm{g} \\
\mathrm{ppm}\end{array}$ & $\begin{array}{l}\text { [Cr] } \\
\mu \mathrm{g} / \mathrm{g} \\
\mathrm{ppm}\end{array}$ & $\begin{array}{l}\text { [Cr] } \\
\mu \mathrm{g} / \mathrm{g} \\
\mathrm{ppm}\end{array}$ & $\begin{array}{l}\text { [Pb] } \\
\mu \mathrm{g} / \mathrm{g} \\
\mathrm{ppm}\end{array}$ & $\begin{array}{l}\text { [Pb] } \\
\mu \mathrm{g} / \mathrm{g} \\
\mathrm{ppm}\end{array}$ & $\begin{array}{l}\text { [Cd] } \\
\mu \mathrm{g} / \mathrm{g} \\
\mathrm{ppm}\end{array}$ & $\begin{array}{l}\text { [Cd] } \\
\mu \mathrm{g} / \mathrm{g} \\
\mathrm{ppm}\end{array}$ \\
\hline RS & Referência & Músculo & < L. D. & 37,090 & 2,170 & 23,730 & 0,360 & 2,810 & 0,110 & 0,120 & $<$ L. D. & 0,230 & $<$ L. D. & 0,020 \\
\hline RS & Referência & Fígado & $<$ L. D. & 38,460 & 4,890 & 90,650 & 3,540 & 20,420 & $<$ L. D. & 0,060 & 0,420 & 0,440 & 0,090 & 0,270 \\
\hline RS & Contaminado & Músculo & 84,120 & 77,750 & 2,040 & 40,520 & 0,260 & 5,640 & 0,200 & 0,160 & $<$ L. D. & 0,200 & $<$ L. D. & 0,020 \\
\hline RS & Contaminado & Fígado & $<$ L. D. & 156,170 & 4,920 & 137,360 & 3,100 & 21,340 & 0,160 & 1,650 & 0,020 & 1,260 & 0,120 & 0,200 \\
\hline PR & Referência & Músculo & 221,470 & 258,980 & 24,730 & 31,160 & 1,470 & 1,650 & 0,160 & 0,040 & 0,410 & 0,500 & 0,010 & 0,030 \\
\hline PR & Referência & Fígado & 989,140 & 724,930 & 784,710 & 490,900 & 160,990 & 56,000 & 0,080 & 1,310 & 1,820 & 3,420 & 1,090 & 2,060 \\
\hline PR & Contaminado & Músculo & 368,120 & 225,020 & 21,750 & 32,310 & 1,840 & 1,720 & 0,530 & $<$ L. D. & 0,210 & 0,210 & 0,010 & 0,040 \\
\hline PR & Contaminado & Fígado & 147,060 & 719,680 & 382,420 & 748,700 & 14,960 & 94,460 & $<$ L. D. & 0,290 & 2,580 & 2,070 & 0,730 & 1,060 \\
\hline ES & Referência & Músculo & $<$ L. D. & $<$ L. D. & 17,060 & 16,470 & 0,770 & 0,610 & 0,100 & 0,110 & 0,260 & 0,600 & $<$ L. D. & 0,080 \\
\hline ES & Referência & Fígado & & $<$ L. D. & & 125,480 & & 12,700 & & 0,270 & & 3,340 & & 0,130 \\
\hline ES & Contaminado & Músculo & $<$ L. D. & $<$ L. D. & 15,950 & 17,170 & 17,420 & 0,690 & 0,490 & $<$ L. D. & 0,410 & 0,460 & 0,420 & 0,020 \\
\hline ES & Contaminado & Fígado & & $<$ L. D. & & 18,160 & & 0,680 & & 0,030 & & 0,490 & & 0,020 \\
\hline PE & Referência & Músculo & & & & & & & & & & & & \\
\hline PE & Referência & Fígado & & & & & & & & & & & & \\
\hline PE & Contaminado & Músculo & 4206,310 & 3379,680 & 17,790 & 23,750 & 2,210 & 1,670 & 0,120 & 0,250 & 0,530 & 0,170 & 0,010 & $<$ L. D. \\
\hline PE & Contaminado & Fígado & 16072,110 & 12594,210 & 1123,010 & 471,620 & 121,180 & 21,420 & 0,220 & 0,130 & 1,900 & 0,710 & 0,360 & 0,120 \\
\hline
\end{tabular}

\begin{tabular}{|c|c|c|c|c|c|c|c|c|c|c|c|c|}
\hline $\begin{array}{l}\text { "LEGISLAÇÃO } \mu \mathrm{g} / \mathrm{g} \\
\text { (PESO ÚMIDO) }\end{array}$ & 0,500 & 0,500 & 50,000 & 50,000 & 30,000 & 30,000 & 0,100 & 0,100 & 2,000 & 2,000 & 1,000 & 1,000 \\
\hline $\begin{array}{l}\text { *LEGISLAÇÃO } \mu \mathrm{g} / \mathrm{g} \\
\text { (PESO SECO) }\end{array}$ & 0,900 & 0,900 & 90,000 & 90,000 & 54,000 & 54,000 & 0,180 & 0,180 & 3,600 & 3,600 & 1,800 & 1,800 \\
\hline
\end{tabular}

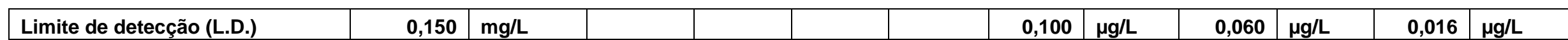

Tabela 2 - Concentrações de metais traço obtidas em Agosto de 2003 e Fevereiro de 2004 em craca (Balanus improvisus) no RS e ostra (Crassostrea rhizophorae) no PR, ES e PE. 
NIENCHESKI, Luis Felipe, et al. Metais traço em peixes e filtradores em quatro estuários da costa brasileira.

\begin{tabular}{|c|c|c|c|c|c|c|c|c|c|c|c|c|c|c|}
\hline \multicolumn{3}{|c|}{ FILTRADORES } & $\begin{array}{l}\text { Ago } \\
2003\end{array}$ & $\begin{array}{c}\text { Fev } \\
2004\end{array}$ & $\begin{array}{c}\text { Ago } \\
2003\end{array}$ & $\begin{array}{c}\text { Fev } \\
2004\end{array}$ & $\begin{array}{c}\text { Ago } \\
2003\end{array}$ & $\begin{array}{c}\text { Fev } \\
2004\end{array}$ & $\begin{array}{l}\text { Ago } \\
2003\end{array}$ & $\begin{array}{l}\text { Fev } \\
2004\end{array}$ & Ago & Fev & Ago & Fev \\
\hline ESTADO & PONTO & MATRIZ & $\begin{array}{l}{[\mathrm{Hg}]} \\
\mathrm{ng} / \mathrm{g} \\
\mathrm{ppb}\end{array}$ & $\begin{array}{l}{[\mathrm{Hg}]} \\
\mathrm{ng} / \mathrm{g} \\
\mathrm{ppb}\end{array}$ & $\begin{array}{l}{[\angle \mathrm{n}]} \\
\mu \mathrm{g} / \mathrm{g} \\
\mathrm{ppm}\end{array}$ & $\begin{array}{l}{[\mathrm{Zn}]} \\
\mu \mathrm{g} / \mathrm{g} \\
\mathrm{ppm}\end{array}$ & $\begin{array}{l}{[\mathrm{Cu}]} \\
\mu \mathrm{g} / \mathrm{g} \\
\mathrm{ppm}\end{array}$ & $\begin{array}{l}{[\mathrm{Cu}]} \\
\mu \mathrm{g} / \mathrm{g} \\
\mathrm{ppm}\end{array}$ & $\begin{array}{l}{[\mathrm{Cr}]} \\
\mu \mathrm{g} / \mathrm{g} \\
\mathrm{ppm}\end{array}$ & $\begin{array}{l}{[\mathrm{Cr}]} \\
\mu \mathrm{g} / \mathrm{g} \\
\mathrm{ppm}\end{array}$ & $\begin{array}{l}{[\mathrm{Pb}]} \\
\mu \mathrm{g} / \mathrm{g} \\
\mathrm{ppm}\end{array}$ & $\begin{array}{l}{[\mathrm{Pb}]} \\
\mu \mathrm{g} / \mathrm{g} \\
\mathrm{ppm}\end{array}$ & $\begin{array}{l}{[\mathrm{Cd}]} \\
\mu \mathrm{g} / \mathrm{g} \\
\mathrm{ppm}\end{array}$ & $\begin{array}{l}{[\mathrm{Cd}]} \\
\mu \mathrm{g} / \mathrm{g} \\
\mathrm{ppm}\end{array}$ \\
\hline RS & Referência & Craca & $<$ L. D. & 142,140 & 810,950 & 1467,210 & 25,360 & 28,520 & 3,610 & 8,290 & 20,210 & 1,650 & 0,930 & 1,250 \\
\hline RS & Contaminado & Craca & 116,060 & 174,920 & 2129,980 & 3756,670 & 48,090 & 47,820 & 7,610 & 3,830 & 6,490 & 35,540 & 1,990 & 2,460 \\
\hline PR & Referência & Ostra & 112,380 & 94,300 & 1754,420 & 2592,790 & 32,290 & 28,190 & 0,550 & 0,580 & 0,240 & 0,160 & 1,510 & 2,040 \\
\hline PR & Contam 1 & Ostra & 168,700 & & 3430,670 & & 54,050 & & 1,240 & & 0,730 & & 1,490 & \\
\hline PR & Contam 2 & Ostra & & 117,990 & & 3581,010 & & 27,030 & & 13,240 & & 0,130 & & 1,160 \\
\hline PR & Contam 4 & Ostra & 88,120 & 63,910 & 3607,150 & 3241,760 & 79,100 & 48,040 & 0,750 & 0,570 & 0,580 & 0,550 & 1,120 & 1,580 \\
\hline ES & Referência & Ostra & $<$ L. D. & $<$ L. D. & 33,540 & 1858,410 & 0,790 & 12,490 & 0,100 & 0,970 & 0,410 & 0,400 & $<$ L. D. & 0,500 \\
\hline ES & Contam 1 & Ostra & $<$ L. D. & & 2434,720 & & 28,520 & & 0,400 & & 0,370 & & 0,490 & \\
\hline ES & Contam 2 & Ostra & & $<$ L. D. & & 1544,700 & & 17,410 & & 0,500 & & 0,550 & & 0,620 \\
\hline ES & Contam 4 & Ostra & 87,900 & $<$ L. D. & 1378,640 & 1459,940 & 25,760 & 21,600 & 0,540 & 1,200 & 0,520 & 0,520 & 0,660 & 0,660 \\
\hline PE & Referência & Ostra & 1330,830 & 652,880 & 912,070 & 1008,770 & 20,230 & 22,790 & 0,320 & 0,980 & 0,610 & 0,410 & 0,870 & 0,620 \\
\hline PE & Contam 1 & Ostra & 1798,240 & & 1243,420 & & 26,780 & & 0,300 & & 0,440 & & 1,070 & \\
\hline PE & Contam 2 & Ostra & & 1405,520 & & 1502,020 & & 22,170 & & 0,780 & & 0,270 & & 1,220 \\
\hline PE & Contam 4 & Ostra & 1600,320 & $<$ L. D. & 1769,930 & 215,840 & 41,810 & 2,440 & 0,450 & 0,030 & 0,640 & 0,150 & 0,910 & $<$ L. D. \\
\hline
\end{tabular}

\begin{tabular}{|c|c|c|c|c|c|c|c|c|c|c|c|c|}
\hline $\begin{array}{l}\text { *LEGISLAÇÃO } \mu \mathrm{g} / \mathrm{g} \text { (PESO } \\
\text { ÚMIDO) }\end{array}$ & 0,500 & 0,500 & 50,000 & 50,000 & 30,000 & 30,000 & 0,100 & 0,100 & 2,000 & 2,000 & 1,000 & 1,000 \\
\hline *LEGISLAÇÃO $\mu \mathrm{g} / \mathrm{g}$ (PESO SECO) & 0,900 & 0,900 & 90,000 & 90,000 & 54,000 & 54,000 & 0,180 & 0,180 & 3,600 & 3,600 & 1,800 & 1,800 \\
\hline Limite de detecção (L.D.) & 0,150 & mg/L & & & & & 0,100 & $\mu g / L$ & 0,060 & $\mu g / L$ & 0,016 & $\mu g / L$ \\
\hline
\end{tabular}


Cobre: mais uma vez, as amostras de fígado, no Paraná, apresentaram concentrações acima do permitido, tanto no inverno como no verão. Amostras de fígado coletadas em Pernambuco, na campanha de agosto, também apresentaram valores acima do limite máximo.

Cromo: em Rio Grande, Paraná, Espírito Santo e Pernambuco ocorreram concentrações maiores que o permitido, tanto no verão como no inverno, no músculo e fígado de peixes coletados em locais de referência e poluído. Exceção ocorreu em Rio Grande, onde as concentrações acima do limite foram encontradas somente no local poluído, coincidindo com o já apresentado por Corradi (2002) e Rosa (2002), onde são citadas as fontes para concentrações elevadas de cromo no Saco da Mangueira.

Zinco: em todos os locais investigados as concentrações também se apresentaram elevadas, nos períodos chuvoso e seco, mas unicamente para fígado (Figura 1).

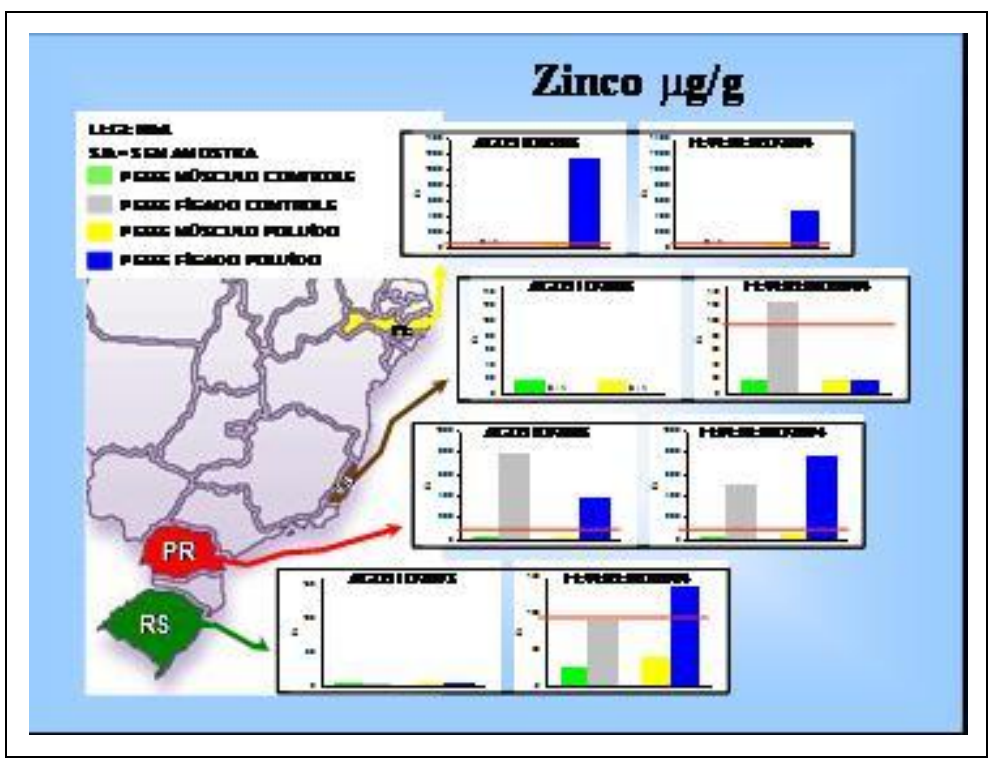

Figura 1 - Concentrações de zinco $(\mu \mathrm{g} / \mathrm{g})$ bioacumulados por peixes em período seco e chuvoso nos quatro estuários avaliados.

Mercúrio: somente Pernambuco, na estação poluída, nas duas campanhas, apresentou concentrações acima do limite máximo, para músculo e fígado (Figura 2).

Em 1996, a descarga total de mercúrio inorgânico oriundo de uma fabrica de produção de cloro e soda cáustica, que opera nas proximidades do rio Botafogo, até 1987, foi estimada por U. Meyer como sendo na ordem de 22 a 35 toneladas e que a descarga atualmente está estimada em 0,03 toneladas/ano, pois a partir de 1986, as operações foram confinadas em uma bacia de precipitação, a qual só passou a operar em sua total capacidade em 1991. Em 1998, Meyer et al realizaram extensa pesquisa sobre o estado da contaminação do Canal de Santa Cruz, sendo analisados material particulado, ostras e sedimentos. Nessa ocasião, os autores concluíram que o rio Botafogo e adjacências foram às áreas mais poluídas por mercúrio. Eles hipotetizam que a maior parte do mercúrio perdido pela fábrica não haveria sedimentado nos ambientes de mangue, mas teria sido exportado ao mar através do material particulado, estando a quantidade presente nos sedimentos entre 1,2 e 2,5 toneladas de mercúrio.

Entretanto, em 2000, Sant Anna et al avaliaram o nível de contaminação por mercúrio total e metil mercúrio em peixes Mugilideos (tainhas) do Canal de Santa Cruz e indicaram que as concentração de mercúrio nos músculos dos peixes mantinha-se na faixa de limites normais e seguros para consumo humano. Mas, em 2003, o relatório divulgado pelo Serviço Geológico do Brasil (CPRM), revelou que o nível de mercúrio do Canal de Santa Cruz estava acima do permitido, tendo sido encontrado 1,1 gramas de mercúrio por tonelada de sedimento do estuário, cerca de $10 \%$ superior ao teor máximo estabelecido pelos órgãos reguladores. Foram analisadas 104 amostras do sedimento do Canal de Santa Cruz, entre Itapissuma e Itamaracá, 
de onde se extrai cerca de $70 \%$ dos peixes, moluscos e crustáceos pescados artesanalmente em Pernambuco.

Para o estuário da Lagoa dos Patos, Niencheski et al. (2001) já haviam demonstrado que as concentrações de mercúrio bioacumulado encontravam-se em níveis abaixo do valor recomendado pela legislação.

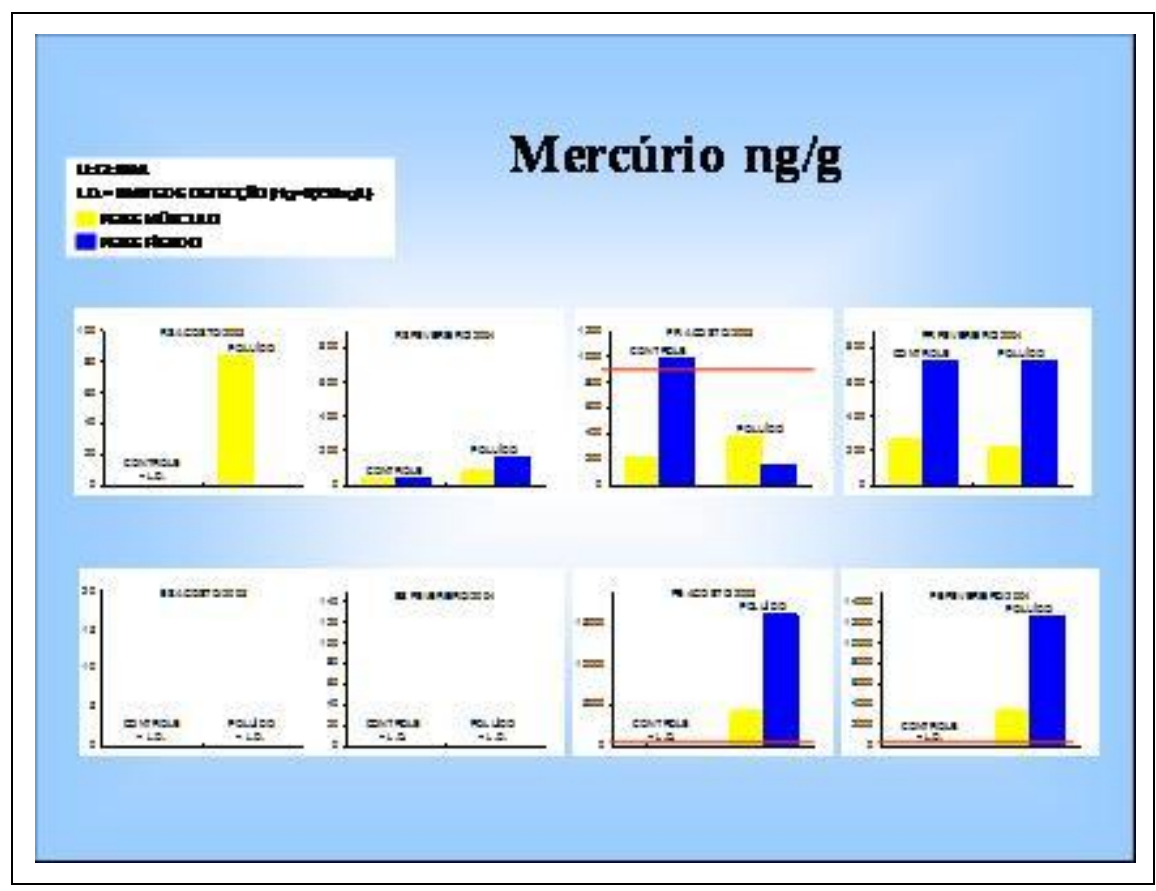

Figura 2 - Concentrações de mercúrio $(\mu \mathrm{g} / \mathrm{g})$ bioacumulados por peixes em período seco e chuvoso nos quatro estuários avaliados.

\section{FILTRADORES}

Chumbo: somente as amostras da Lagoa dos Patos apresentaram concentrações de chumbo acima dos limites máximos recomendados pela legislação. Concentrações elevadas de chumbo e os processos envolvidos em seu transporte nesse estuário são discutidas em Baraj et al. (2003) e Niencheski et al. (2004).

Cádmio: novamente no estuário da Lagoa dos Patos, nas duas campanhas (local poluído) e na Baía de Paranaguá (local referencia) na segunda campanha, apresentaram concentrações ligeiramente acima do limite máximo. No caso do RS, as concentrações de cádmio encontradas no Saco da Mangueira (local poluído) podem ser o resultado de lançamentos associados com a atividade das indústrias de fertilizante, ao longo dos últimos 25 anos (Baumgarten, 1987). Relativas altas concentrações de $\mathrm{Cd}$ tem sido encontradas na matéria prima usada na produção de fertilizantes, resultando em efluentes líquidos e aéreos contaminados (Furley, 1993 e Niencheski et al., 2004).

Cobre: somente a estação poluída da Baía de Paranaguá apresentou destaque quanto à concentração fora dos limites estabelecidos pela ABIA (1991).

Cromo: todos os locais, nas duas campanhas apresentaram concentrações que se situaram acima do permitido (Figura 3). Exceção foi no estuário dos rios Piraquê-Açu e PiraquêMirim (ES) na estação referencia (primeira campanha) e Pernambuco na estação poluída (segunda campanha). Entretanto, é de causar questionamento o fato da estação poluída de Pernambuco apresentar concentrações abaixo do limite. No RS, as concentrações elevadas não se constituem em algo desconhecido e, aplicam-se aqui os comentários apresentados para as concentrações bioacumuladas nos peixes.

Zinco: todos os locais investigados também apresentaram concentrações elevadas, nas duas campanhas amostrais, com uma única exceção que foi a estação referência de Vitória, na primeira campanha amostral. O zinco apresentou comportamento bastante semelhante ao 
cromo, o que indica que esses metais tenham fontes de aporte comuns e, que deveriam ser investigadas no futuro.

Mercúrio: semelhante aos peixes, somente em Pernambuco, as concentrações foram acima do limite máximo. Definitivamente, o mercúrio é um problema para o Complexo Estuarino do Canal de Santa Cruz, conforme resultados pretéritos (Meyer, 1996), Meyer et al.(1998), Sant`Anna et al. (2000) e CPRM (2003).

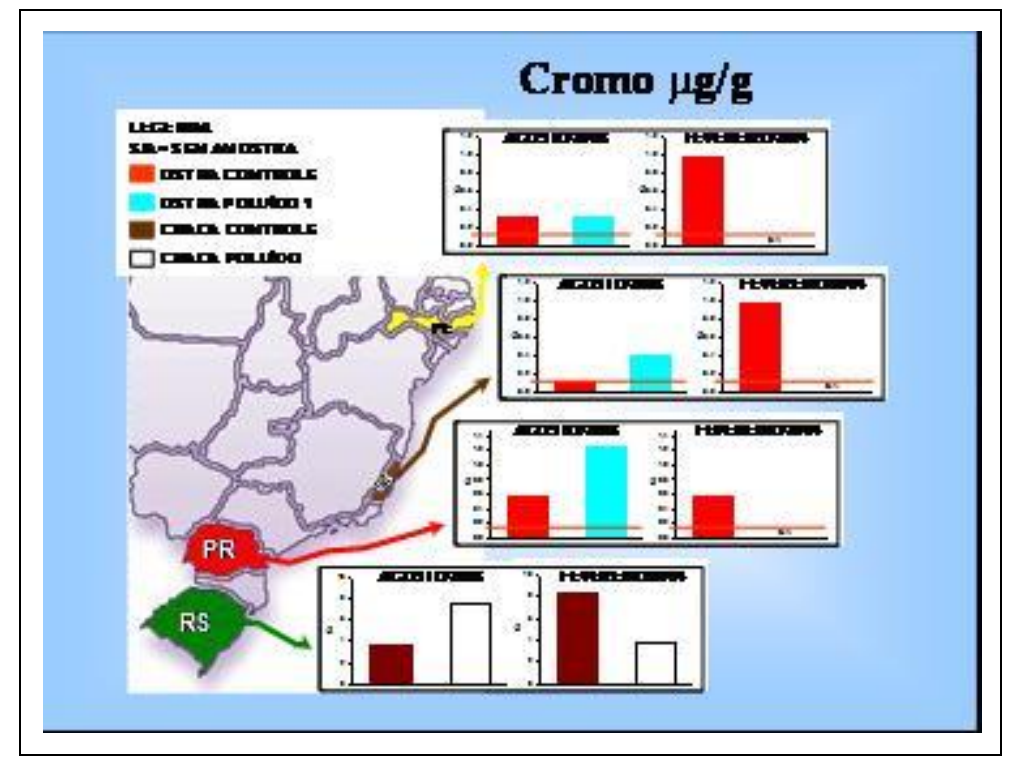

Figura 3 - Concentrações de cromo $(\mu \mathrm{g} / \mathrm{g})$ bioacumulados por cracas em período seco e chuvoso nos quatro estuários avaliados.

\section{CONCLUSÃO}

Peixes, ostras e cracas analisados apresentaram concentrações de metais traço acima do limite máximo recomendado pela legislação brasileira em alimentos para consumo humano. Alguns metais exibiram concentrações que excederam os máximos permitidos em quase todas as amostras analisadas, revelando a necessidade urgente de um estudo sistemático sobre a bioacumulação destes metais e, também sobre a forma como eles se apresentam no meio ambiente (estudos de especiação química).

A detecção de concentrações acima do limite máximo estipulado pela legislação brasileira, nos locais denominados poluídos confirmaram as hipóteses do Grupo QA\&B, de que as fontes de poluição existentes nesses locais afetam a qualidade da biota. Entretanto, causa preocupação as concentrações elevadas nos locais considerados referência.

Recomenda-se fortemente que programas de monitoramento espacial e temporal sejam aplicados para geração de dados de base continuados, os quais acoplados a dados oceanográficos/estuarinos e associados ao conhecimento dos processos envolvidos tanto naturais como antrópicos (considera-se aqui a importância do conhecimento das fontes potenciais e quantificação do lançamento de metais em cada região), forneçam a base necessária para a elaboração de balanços de massa, para um posterior estudo da contaminação e poluição. De forma alguma esses estudos podem ficar dissociados de uma integração profunda com os órgãos de fiscalização/normatização, para que seja atingido o objetivo final que é a redução da poluição e restauração da "saúde" do ambiente.

Com esse trabalho, foram lançadas as bases para um programa de monitoramento, pois: i) buscou informações de base e promoveu treinamento de equipes em estudos piloto, planejamento e execução, ii) estabeleceu um desenho do programa de monitoramento, iii) promoveu a seleção de contaminantes, de espécies e de tecidos biológicos e, iv) padronizou procedimentos analíticos. Em conjunto, tais ações permitem que sejam mais bem conhecidas as relações da biota com as características físicas e químicas do ambiente e com os produtores 
primários e secundários, incluindo as interferências humanas como o principal fator de alteração das condições naturais.

\section{AGRADECIMENTOS}

Aos técnicos químicos Edi Morales Pinheiro Junior, José Vanderlen Veigas Miranda e Lúcia Helena Bohmer e, demais componentes do Laboratório de Hidroquimica pelo valioso apoio e participações nas amostragens e nas análises.

\section{REFERÊNCIAS BIBLIOGRÁFICAS}

ABIA (Associação Brasileira das Indústrias de Alimentação). Compêndio da Legislação de Alimentos. Atos do Ministério da Saúde. 1991.

ANGELI, J. L. F. Elementos traço em peixes do Complexo Estuarino de Paranaguá. Pontal do Sul, 2012. 29 p. Dissertação (Mestrado em PGSISCO - Sistemas Costeiros e Oceânicos). Universidade Federal do Paraná.

ANVISA. 1998. Legislação Brasileira, Portaria 685. Disponível em

http://www.anvisa.gov.br/legis/portarias/685_98.htm. Acessado em 30 Agosto de 2011.

Avaliação Ambiental de Estuários Brasileiros: Diretrizes Metodológicas (2006). Org. Lana, P.C. et al. Rio de Janeiro, Museu Nacional. 156 p. ISBN 85-7427-017-2.

BARAJ, B.; NIENCHESKI, L.F. \& CORRADI, C. Trace metal content trend of mussel Perna perna from the Atlantic coast of southern Brazil. Water, Air, and Soil Pollution. 145 (1-4), p. 205214. 2003. ISSN 0049-6079.

BARROSO, G.F. Assessing the potential for mangrove oyster aquaculture in an estuarine system of the southeastern coast of Brazil: A geographic information system approach. Tese de Doutorado em Geografia. 2004. University of Victoria, UVic, Canadá.

BAUMGARTEN, M. G. Z. Avaliação do Balanus improvisus como indicador dos níveis metálicos do estuário da Lagoa dos Patos (RS-Brasil). Rio Grande, 1987. 178 p. Dissertação de Mestrado. FURG.

BAUMGARTEN, M.G.Z.; NIENCHESKI, L.F. \& KUROSHIMA, K.N.. Qualidade das águas estuarinas que margeiam o município do Rio Grande (RS): Nutrientes e detergente dissolvidos. Revista Atlântica. Vol. 17: 17-34. 1995.

BAUMGARTEN, M.G.Z.; NIENCHESKI, L.F. \& VEECK, L. Nutrientes na coluna d'água e na água intersticial de sedimentos de uma enseada rasa estuarina com aportes antrópicos (RS - Brasil). Revista Atlântica. Volume 23. p. 101 - 116. 2001.

CASTELLO, B. F. L. Avaliação dos teores de As, Cu, Cd, Ni e Zn em ostras, Crassostrea rhizophorae (Guilding, 1828), nas baías de Paranaguá e Guaratuba, Paraná. Pontal do Sul, 2011. 67p. Dissertação (Mestrado em PGSISCO - Sistemas costeiros e oceânicos) Universidade Federal do Paraná.

CHOUERI, R.B.; CESAR, A.; TORRES, R.J.; ABESSA, D.M.S.; MORAIS, R.D.; PEREIRA, C.D.S.; NASCIMENTO, M.R.L.; MOZETO, A.A.; RIBA, I.; DELVALLS, T.A. 2009. Integrated sediment quality assessment in Paranaguá Estuarine System, Southern Brazil. Ecotoxicology and Environmental Safety, v. 72, p. 1824-1831. 2009.

C.P.R.H. Monitoramento de Bacias Hidrográficas do Estado de Pernambuco 2004, Recife, 2004.

C.P.R.M. Sistema de informações geoambientais da Região Metropolitana do Recife. Recife, Companhia de Pesquisa de Recursos Minerais, P.A.S. Pfaltzgraff (Coord.), 2003. 119 p., (CD).

CORRADI, C. E. Avaliação das condições do litoral do Rio Grande do Sul através da bioacumulação de metais pesados por mexilhões e, por cracas e camarões no estuário da Lagoa dos Patos. Rio Grande, 2002. 82p. Dissertação de Mestrado em Oceanografia Biológica. FURG. 
FILLMANN, G.; MACHADO, E. C.; MARTINS, C. C.; SÁ, F. Poluentes orgânicos persistentes nos sedimentos dos canais de acesso aos portos de Paranaguá e Antonina (PR). In: Boldrini, E. B.; Soares, C. R.; de Paula, E. V.. (Org.). Dragagens portuárias no Brasil: licenciamento e monitoramento ambiental. 1a ed. Curitiba: Josimar Marchiorato, v. 1, p. 264275. 2007.

FROEHNER, S.; MACENO, M.; MACHADO, K. S. Predicting bioaccumulation of PAHs in the trophic chain in the estuary region of Paranagua, Brazil. Environmental Monitoring and Assessment (Print), v. 174, p. 135-145. 2011.

FURLEY, T. H. Utilização do mexilhão Perna perna (Linné, 1758) como bioindicador dos metais pesados cádmio, chumbo, zinco, cobre e manganês do litoral do Rio Grande do Sul (Brasil). Rio Grande, 1993. 131 p. Dissertação de Mestrado em Oceanografia Biológica. FURG.

IBGE 2010. Censo 2010. Instituto Brasileiro de Geografia e Estatística. Ministério do Planejamento, Orçamento e Gestão. http://www.ibge.gov.br.

LIEBEZEIT, G.; BREPOHL, D.; RIZZI, J.; GUEBERT, F.; KROME, M.; MACHADO, E.; PIJANOWSKA, U. DDT in Biota of Paranaguá Bay, Southern Brazil: Recent Input and Rapid Degradation. Water, Air and Soil Pollution (Print), p. 2137-2143. 2011.

MARTINS, C.C.; ANGELLI, J.L.F.; COMBI, T.; GALLICE, W.C.; MANSUR, A.V.; NARDES, E.; ROCHA, M.L.; WISNIESKI, E.; CESCHIM, L.M.M.; RIBEIRO, A.P. Multi-molecular markers and metals as tracers of organic matter inputs and contamination status from an Environmental Protection Area in the SW Atlantic (Laranjeiras Bay, Brazil). Science of the Total Environment, v. 417-418, p. 158-168. 2012.

MARTINS, C.C.; BRAUNS, J.A.F; SEYFFERT, B.H.; MACHADO, E.C.; FILLMANN, G. Anthropogenic organic matter inputs indicated by sedimentary fecal steroids in a large South American tropical estuary (Paranaguá estuarine system, Brazil). Marine Pollution Bulletin. p. 2137-2143, 2010.

M. E. S. L.; I. A. E. A. Standard Operating Procedures, Monaco, 85 p. 1997.

MEYER, U. On the fate of mercury in the northeastern brazilian mangrove system, Canal de Santa Cruz, Pernambuco. ZMT - Contributions 3, Bremen. Alemanha. 1996.

MEYER, U. HAGEM, W.; MEDEIROS, C. Mercury in a Northeastern Brasilian Mangrove Area, a case study: Potencial of the Mangrove oyster Crassostrea rhizophorae as Bioindicator for Mercury. Marine Biology, v. 131, p. 113-121. 1998.

NIENCHESKI, L.F., H.L. WINDOM, B. BARAJ, D. WELLS \& R. SMITH. Mercury in Fishes from Patos and Mirim Lagoons, Southern Brazil. Marine Pollution Bulletin. v. 42. No. 12, p. 14031406. 2001.

NIENCHESKI, L.F.; BARAJ, B.; WINDOM, H. \& R. FRANÇA. Background assessment and its anthropogenic contamination of $\mathrm{Cd}, \mathrm{Pb}, \mathrm{Cu}, \mathrm{Cr}, \mathrm{Zn}, \mathrm{Al}$ and $\mathrm{Fe}$ in the sediments of the Southern area of Patos Lagoon. Journal of Coastal Research SI 39, p. 1040 - 1043 ICS 2004 (Proceedings) Brazil ISSN 0749-0208. 2004.

ROSA, M.L. Concentrações de metais pesados na biota e sedimentos do estuário da Lagoa dos Patos. Rio Grande. 2002 244p. Mestrado em Engenharia Oceânica. FURG.

SÁ, F.; MACHADO, E.C.; ANGULO, R.J. ; VEIGA, F.A.; BRANDINI, N. Arsenic and trace metals in sediment near Paranaguá Port. Journal of Coastal Research, v. 39, p. 1066-1068. 2006.

SÁ, F. Distribuição e fracionamento de contaminantes nos sedimentos superficiais e atividades de dragagem no Complexo Estuarino da Baía de Paranaguá (PR). Pontal do Sul. 2003. Dissertação de Mestrado em PGSISCO (Sistemas Costeiros e Oceânicos). Universidade Federal do Paraná.

SANT 'ANNA, N.; COSTA, M.; AKAGI, H. Total and methylmercury levels of the coastal human population and of fish from the Brazilian Northeast. Environmental Science and Pollution Research, v. 8, p.280-284. 2001. 
CASTRO, N. F.; FEITOSA, F. A. do N.; FLORES MONTES, M. de J. Avaliação das condições Ambientais do estuário do rio Carrapicho (Itamaracá- PE): Biomassa fitoplanctônica e Hidrologia.

UNEP/FAO/IAEA/IOC. Determination of total cadmium, zinc, lead and copper in selected marine organisms by flameless atomic absorption spectrophotometry. Reference Methods for Marine Pollution Studies. No. 11. 21p. 1984a.

UNEP/FAO/IAEA/IOC. Determination of total mercury in selected marine organisms by could vapour atomic absorption spectrophotometry. Reference Methods for Marine Pollution Studies. No. 8. 17p. 1984b.

USTRA, J. R. Concentrações de mercúrio na biota e sedimentos no sul do estuário da Lagoa dos Patos. Rio Grande. 2001. 125 p. Dissertação de Mestrado em Engenharia Oceânica. FURG. 